\title{
Is Prefilled Contrast Agent Effective in Timesaving and Patient Safety
}

\author{
Jinhyun Kim ${ }^{1}$, Suyong Jeong ${ }^{1}$, Jiming Tang ${ }^{1}$, Eunhee Lee ${ }^{2 *}$ \\ ${ }^{1}$ College of Nursing, Seoul National University, Korea \\ ${ }^{2}$ Division of Nursing, Hallym University, Korea
}

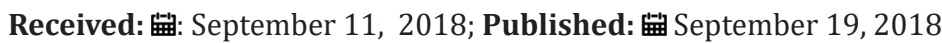

*Corresponding author: Eunhee Lee, Division of Nursing, Hallym University, 24252 Republic of Korea

\begin{abstract}
Background: In CT imaging, when preparing contrast agent, the prefilled type is simpler to use than is the vial type. In this study, the benefits from using prefilled contrast agent were examined.

Methods: The benefits of the prefilled type were defined as time saving and prevention of infection. We measured the preparation time in 3 general hospitals, and then estimated the time saving benefit by multiplying the result by the cost of the CT scan. To gauge infection prevention, we conducted a decision analytic model and estimated the benefits using the probability of infection from the decision model and treatment costs.

Results: The cost of both drugs was equal; however, the additional material cost associated with vial type agent was $\$ 18.18$, which is higher than that of the prefilled type. The reduction in time from using the prefilled type was on average 51.9 seconds, and the time savings was estimated as $\$ 7.22$ per case. The benefit of preventing infection was estimated at $\$ 0.24$ per case when using the prefilled type. Therefore, the total benefit was estimated at $\$ 25.64$. Conclusions: The prefilled type was superior to the vial type in that it was associated with lower costs and higher benefits.
\end{abstract}

Keywords: Contrast Agent; Ct Scan; Time Management; Infection Control

\section{Introduction}

Ultravist has been used as a contrast agent in computed tomography (CT) imaging and allows for an efficient and optimized workflow $[1,2]$. This contrast agent is marketed in two types, which are the vial type and the prefilled type. In the case of vial type contrast agent, for assembly and refilling, the contrast agent must be moved to an automatic injector using syringes, disposable tubes, and connectors. During this process, the staff frequently manipulates the contrast agent and several materials necessary for assembly. The materials that do not come into direct contact with a patient can be used for several examinations or for more than one patient, although the materials used during treatment are approved for single use only according to several guidelines about infection control [3]. Frequent handling and multiple uses of syringe and other materials potentially leads to bacterial contamination and local infection [4-8].

On the other hand, the prefilled contrast agent requires no syringe filling during the assembly procedure. When using the prefilled type, fewer manipulations are done by the staff than are necessary when using vial type contrast agent; therefore, it has a benefit of lowering the risk of contamination or infection. Moreover, as the time required to assemble all the materials is shorter, the procedure is done with greater efficiency and there is a time saving benefit as well [5-9]. Hospital-acquired infections are an important indicator of patient safety, and influence treatment period and cost [6,10-12]. In order to reduce infection, disposable materials such as syringes and connecting tubes should be used only once, even though they do not come into contact with patients directly [3]. Moreover, the Centers for Disease Control and Prevention (CDC) guidelines recommended that single-dose or single-use vials should be used clinically only for one dose and for one patient $[13,14]$.

The contrast agent in a vial could be used in several patients, at the same time, the materials could be used several times as well. It is difficult for hospital staff to ensure that the vial and the materials are kept sterile. Therefore, prefilled contrast agent is more suitable than vial type contrast agent in terms of patient safety. While there have been several studies on the effectiveness, safety, and economic evaluation of various medicines, diseases and operation, there is a dearth of research about the materials or devices used for medical treatment. In particular, material that is closely related with patient safety should be examined for benefits such as efficiency and safety. 
Therefore, this study aimed to evaluate the benefit of the two different contrast agent injection systems, prefilled type and vial type, with regard to infection management and time saving.

\section{Methods}

This study aimed to evaluate the benefits of prefilled contrast agent vs. vial type contrast agent. The prefilled type was found to be superior in terms of time saving and prevention of infection, and the benefits of prefilled contrast agent were defined as an increase in profit and cost saving.

\section{Study Design}

This study was designed as an economic analysis using clinical data, secondary research, and health insurance data from the National Health Insurance (NHI) Corporation [15]. The NHI database includes information on healthcare utilization by the entire population in Korea. To determine treatment cost from NHI data, we calculated the benefit of prefilled contrast agent given its effects on time saving and infection control.

\section{Estimating the Benefit of Prefilled Cartridges}

Use of prefilled contrast agent saves time because it allows hospital staff to skip several steps in the assembly process and prevents infection because it decreases unnecessary handling. To estimate the extent of time saving, we collected data from three hospitals where the two kinds of contrast agents (vial type and prefilled type) were used for CT imaging, and measured the preparation time repeatedly in these 3 general hospitals. As there were few cases of CT imaging using the prefilled type, we identified the assembly procedure of both types and measured the time saved owing to omitted procedures when using the prefilled type. We observed 113 cases in the 3 general hospitals. Assuming that the reduced time was then available for CT scans to be given to other patients, we estimated the time savings by multiplying the result by the cost of a CT scan. The extent of the benefit regarding infection was estimated in 3 steps:

a) conducting a decision analytic model

b) calculating the parameters using secondary research

c) estimating the benefit using a folding-back method.

First, we constructed the decision analytic model to evaluate the effectiveness according to type (Figure 1). The invasive procedure could potentially lead to local infection, such as phlebitis, or a blood stream infection (BSI) in serious cases, regardless of whether the materials are contaminated or not. If the material is contaminated, the rate of infection will be higher. After conducting the decision analytic model, we found the parameters for the decision tree through a literature review. According to a study by Buerke et al., microbiological contamination occurred 3.3\% of the time when materials were used multiple times, but $0.0 \%$ of the time with single-use prefilled contrast agent [16]. As there is a lack of research into the probability of infection to contamination of materials, we referred to the results obtained from similar studies. Owing to materials contamination, the local infection rate was $2.1 \%$ according to a study by Lee el al. [17] and the rate of BSI was $0.1 \%$ according to Maki et al. [8].

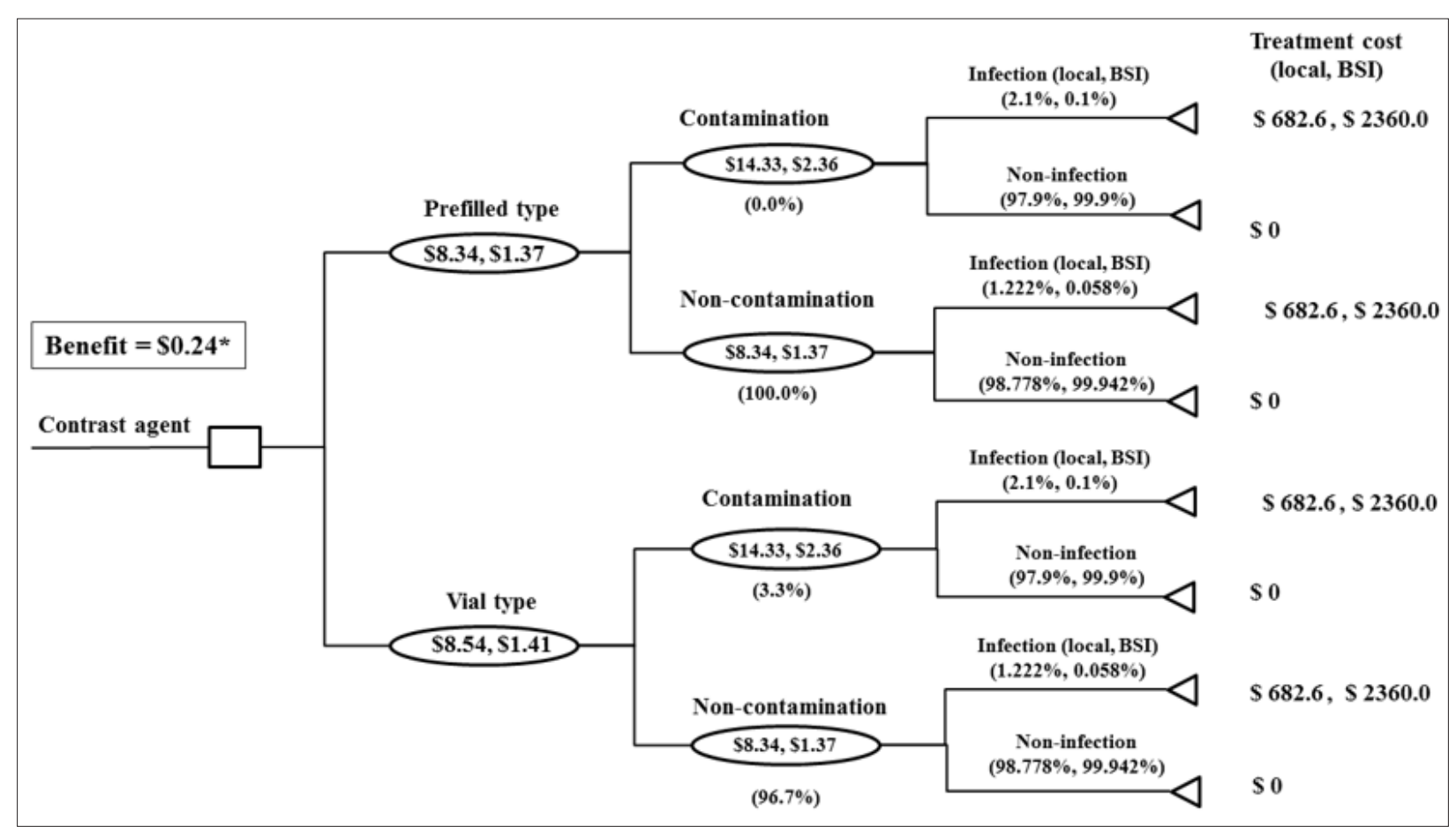

Figure 1: Benefit for preventing infection.

*Benefit was the difference in treatment cost between prefilled typed and vial type. Benefit $(\$ 0.24)=$ local infection benefit $(\$ 8.54-\$ 8.34)+$ BSI benefit $(\$ 1.41-\$ 1.37)$ 
In addition, we calculated the probability of local infection, and BSI was calculated by relative risk (0.582) from the result of a study by Veenstra et al. [8] because there is a lack of research into the probability of infection when using contamination-free material. We then estimated the benefits by folding back, multiplying the probability of infection (local infection and BSI) by the treatment costs for local infections or BSI in 2015 obtained from the NHI database [15].

\section{Sensitivity Analysis}

Sensitivity analysis was conducted to adjust for the uncertainty of reducing time, examination cost, and infection probability, which was estimated by secondary studies. The interval of sensitivity analysis was applied to the mean and standard deviation of the estimated reduction in time, cost of a CT scan according to body part examined, and confidence interval of the secondary research into the probability of infection.

\section{Results}

The cost of vial type and prefilled type contrast agent was initially equal; however, the additional material cost related to the use of vial type agent was $\$ 18.18$, which is higher than the materials cost associated with the prefilled type. Thus, the total cost of the prefilled type was actually lower than that of the vial type. The reduction in time obtained by using prefilled contrast agent was, on average, 51.9 seconds ( \pm 9.6 seconds) based on 113 observations from 3 general hospitals. As the average CT scan cost $\$ 125.12$ and took about 15 minutes, the average cost of CT imaging per second was estimated at $\$ 0.14$. Assuming that the reduced time was used to give CT images to other patients, the benefit of time saving was estimated at $\$ 7.22$ per case (Table 1). In addition, the probabilities of contamination, local infection, and BSI when using vial type contrast agent were $3.300 \%, 1.251 \%$, and $0.060 \%$ respectively, which were higher than those associated with the prefilled type $(0.000 \%, 1.222 \%$, and $0.058 \%$, respectively). The prevention rate of infection due to the use of prefilled type was $0.029 \%$ for local infections and $0.002 \%$ for BSI. Accordingly, the financial benefit from infection control when using prefilled contrast agent was estimated at $\$ 0.24$ per case (Figure 1 ). The total benefit was estimated at $\$ 7.46$, including $\$ 0.24$ in infection control and $\$ 7.22$ in time savings.

Table 1: Reducing time and time saving benefit.

\begin{tabular}{|c|c|}
\hline & Mean \pm SD \\
\hline Reducing time $(\mathrm{n}=113$, seconds) & \\
\hline Average & $51.9 \pm 9.6$ \\
\hline Hospital A (n=90) & $51.0 \pm 9.5$ \\
\hline Hospital B $(\mathrm{n}=16)$ & $56.2 \pm 8.4$ \\
\hline Hospital C $(\mathrm{n}=7)$ & $53.9 \pm 11.9$ \\
\hline Cost for a CT-scan $(\$)$ & \\
\hline Average & $125.12 \pm 0.14$ \\
\hline Brain & $111.24 \pm 0.12$ \\
\hline Chest & $131.65 \pm 0.15$ \\
\hline
\end{tabular}

\begin{tabular}{|c|c|}
\hline Abdomen & $126.87 \pm 0.14$ \\
\hline Spine & $130.70 \pm 0.15$ \\
\hline Time saving benefit $(\$)$ & 7.22 \\
\hline
\end{tabular}

Table 2: Benefit of prefilled type.

\begin{tabular}{|c|c|c|}
\hline Categories & Saving cost $\mathbf{( \$ )}$ & $\mathbf{\%}$ \\
\hline Total benefit per case & $\mathbf{2 5 . 6 4}$ & $\mathbf{1 0 0 . 0}$ \\
\hline Direct cost saving & 18.18 & 70.9 \\
\hline Benefits & 7.46 & 29.1 \\
\hline Time saving & 7.22 & 28.2 \\
\hline Preventing infection & 0.24 & 0.9 \\
\hline
\end{tabular}

Table 3: Sensitivity analysis.

\begin{tabular}{|c|c|}
\hline Sensitivity Analysis & Benefit (\$) \\
\hline Unit cost and reducing time & \\
\hline Unit cost $\$ 0.124$ and reducing time 42.3 seconds & 5.23 \\
\hline Unit cost $\$ 0.139$ and reducing time 51.9 seconds* & 7.22 \\
\hline Unit cost $\$ 0.146$ and reducing time 61.5 seconds & 9 \\
\hline Relative risk & \\
\hline 0.851 & 0.08 \\
\hline $0.582 *$ & 0.24 \\
\hline 0.398 & 0.33 \\
\hline Total benefit & 23.49 \\
\hline Minimum & 25.64 \\
\hline Basecase & 27.51 \\
\hline Maximum & \\
\hline
\end{tabular}

Thus, the total amount saved per case was estimated at $\$ 25.63$, which includes a direct cost saving of $\$ 18.18$ (70.9\%) and a benefit of $\$ 7.46$ (29.1\%) (Table 2). To account for the uncertainty in this study, we performed sensitivity analysis according to the estimated time reduction, cost of CT imaging, and infection rate. The benefit of time saving was estimated at $\$ 5.23 \sim \$ 9.00$ according to the change in reduced time and cost for $\mathrm{CT}$ imaging. In addition, the benefit of infection control was estimated at $\$ 0.08 \sim \$ 0.33$ by the change in relative risk. Finally, the minimum benefit of using the prefilled type was $\$ 23.49$ per case, and the maximum benefit was $\$ 27.51$ per case (Table 3).

\section{Discussion}

This study investigated the economic benefit of a new injection system for contrast agent. The improvement associated with the prefilled type was measured in two parts

a) time saving due to simplification of the assembly process

b) prevention of infection due to avoidance of multiple uses of materials such as syringes, tubes, and connectors.

The reduction in time associated with the use of prefilled contrast agent in this study was $\mathbf{5 1 . 9}$ seconds, which is consistent with the results of other studies, which reported that the reduction does not exceed one minute [5]. The absolute reduction in time per 
case is not long; however, the cumulative time reduction is huge considering the frequency of CT imaging. In one of the hospitals in this study, the number of examinations per CT scan machine was approximately 70 per day. If all cases used the prefilled type, the time saving benefit per day would exceed $\$ 500$ for only a single machine. Therefore, the time saving benefit would be enormous in a large hospital. In addition, the use of the prefilled type is important in terms of patient safety, even though the benefit regarding infection prevention was not huge.

For the vial type contrast agent, the assembly materials, such as injector syringes, tubes, and connectors, were used for several examinations or several patients, despite multiple uses of medical materials being banned in principle [3]. In several hospitals, multiple uses of materials for treatment are happening in everyday practice [4]. Buerke et al. [5] reported that the rate of contamination was $3.3 \%$ when syringes were used multiple times [5]. As the staff in Korea often used an injector syringe many times, the rate of contamination is probably higher than that reported in Buerke et al.'s study [5], and the high rate of contamination most likely resulted in an increased incidence of severe infection $[7,8,19,20]$ In addition, it is well known that the multi-dose vials (MDV), such as those containing heparin and insulin, are extremely vulnerable to contamination $[13,14,21]$. Contaminated MDVs are responsible for $80 \%$ of nosocomial infections, and, in those cases, the mortality rate is $10 \%$ [21].

In CT imaging, vial type contrast agent may be used for some patients according to circumstances. Hence, the risk of infection due to the use of vial type contrast agent might be greater than expected. In order to estimate the benefit regarding prevention of infection, we performed a literature review; however, there was no previous study about the infection rate associated with prefilled contrast agent. Therefore, we estimated the infection prevention benefit through a decision tree model. The benefit due to infection prevention was estimated at $\$ 0.23$, which accounted for only $0.9 \%$ of the total benefit. Considering the frequency of the use of injector syringes in Korea, the infection rate and the costs associated with infection might be higher in real practice than those suggested by the results of this study. Besides the time saving and infection prevention benefits, the prefilled type might have a benefit regarding waste disposal and storage of medicines or materials.

However, we excluded these benefits from our analysis because of uncertainty given the limited amount of data available. Therefore, further research is needed to estimate the costs incurred during the entire process of CT imaging, including storage, preparation, and disposal. Prefilled type contrast agent had benefits regarding time saving and infection prevention, and the direct cost of prefilled contrast agent is lower than that of vial type contrast agent. The cost of prefilled type equates to that of vial type in Korea, while the cost of prefilled type is higher than vial type in several other countries. Therefore, according to the price of the prefilled type, the benefit could be lower than the result in our study. However, as the overall trend has been that hospitals gradually reinforce the regulations regarding infection control $[3,13]$, the prefilled type should be recommended for use in CT imaging, if only for the simple reason that the prefilled type is safer than the vial type.

The materials necessary for treatment have scarcely been evaluated for their effectiveness or safety, compared to the medicine itself. However, among the various materials, there are some that had as strong an influence on patient safety as the medicines. Thus, the effect of the materials on patient safety should be further evaluated in term of safety. Contrast agent is injected directly into blood vessels; therefore, the procedure could give rise to serious infections in cases of contamination of materials or contrast agent. In order to protect patient safety, we need to use the safer method for CT imaging.

\section{Conclusion}

The present results showed that the prefilled type of contrast agent dominated the vial type in terms of lower costs and higher benefits. This was driven by lower materials cost, lower infection risk, and reduced administration time for the prefilled type compared to the vial type. In addition, given that syringes are reused several times in Korea, we expect that the financial benefit associated with infection control is in fact greater than $\$ 0.23$. The findings of this study suggest that the use of PFC contrast agent is an efficient utilization of resources in CT imaging.

\section{Acknowledgment}

This work was supported by Bayer Korea and Hallym University (HRF-201706-014).

\section{References}

1. Weininger M, Barraza JM, Kemper CA, Kalafut JF, Costello P, et al. (2011) Cardiothoracic CT angiography: current contrast medium delivery strategies. AJR American journal of roentgenology 196: W260-W272.

2. Fleischmann D, Kamaya A (2009) Optimal vascular and parenchymal contrast enhancement: the current state of the art. Radiologic clinics of North America 47(1): 13-26.

3. Yokoe DS, Anderson DJ, Berenholtz SM, Calfee DP, Dubberke ER, et al. (2014) A compendium of strategies to prevent healthcare-associated infections in acute care hospitals: 2014 updates. Infection control and hospital epidemiology 35(8): 967-977.

4. Buerke B, Mellmann A, Stehling C, Wessling J, Heindel W, et al. (2008) Microbiologic contamination of automatic injectors at MDCT: experimental and clinical investigations. AJR American journal of roentgenology 191: W283-W287.

5. Buerke B, Puesken M, Mellmann A, Seifarth H, Heindel W, et al. (2010) Microbiologic contamination and time efficiency of use of automatic MDCT injectors with prefilled syringes: Results of a clinical investigation. AJR American journal of roentgenology 194(2): 299-303.

6. Pujol M, Hornero A, Saballs M, Argerich MJ, Verdaguer R, et al. (2007) Clinical epidemiology and outcomes of peripheral venous catheterrelated bloodstream infections at a university-affiliated hospital. The Journal of hospital infection 67(1): 22-29.

7. Weintraub AM, Ponce de Leon MP (1998) Potential for crosscontamination from use of a needleless injector. American journal of infection control 26(4): 442-445.

8. Maki DG, Kluger DM, Crnich CJ (2006) The risk of bloodstream infection in adults with different intravascular devices: a systematic review of 200 published prospective studies. Mayo Clinic proceedings 81(9): 11591171. 
9. Veenstra DL, Saint S, Saha S, Lumley T, Sullivan SD (1999) Efficacy of antiseptic-impregnated central venous catheters in preventing catheterrelated bloodstream infection: a meta-analysis. Jama 281(3): 261-267.

10. Gandra S, Barysauskas CM, Mack DA, Barton B, Finberg R, et al. (2014) Impact of contact precautions on falls, pressure ulcers and transmission of MRSA and VRE in hospitalized patients. The Journal of hospital infection 88(3): 170-176.

11. Edmond MB, Masroor N, Stevens MP, Ober J, Bearman G (2015) The Impact of Discontinuing Contact Precautions for VRE and MRSA on Device-Associated Infections. Infection control and hospital epidemiology 36(8): 978-980.

12. Saint S, Veenstra DL, Lipsky BA (2000) The clinical and economic consequences of nosocomial central venous catheter-related infection: are antimicrobial catheters useful? Infection control and hospital epidemiology 21(6): 375-380.

13. (2012) Centers for Disease Ccontol Prevention. Invasive Staphylococcus aureus infections associated with pain injections and reuse of singledose vials--Arizona and Delaware. MMWR Morbidity and mortality weekly report 61(27): 501-504.

14. Manchikanti L, Falco FJ, Benyamin RM, Caraway DL, Helm Ii S, et al. (2012) Assessment of infection control practices for interventional techniques: a best evidence synthesis of safe injection practices and use of single-dose medication vials. Pain physician 15(5): E573-E614.

\section{ISSN: 2574-1241}

DOI: $10.26717 / B J S T R .2018 .09 .001759$

Eunhee Lee. Biomed J Sci \& Tech Res

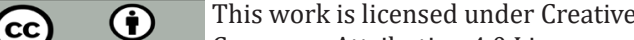
Commons Attribution 4.0 License

Submission Link: https://biomedres.us/submit-manuscript.php
15. (2015) Korea Heatlh Insurance Cooperation. Health insurance statistical yearbook. Seoul: National Health Insurance Cooperation, Korea.

16. Buerke B, Puesken M, Mellmann A, Schuelke C, Knauer A, et al. (2011) Automatic MDCT injectors: hygiene and efficiency of disposable, prefilled, and multidosing roller pump systems in clinical routine. AJR American journal of roentgenology 197(2): W226-W232.

17. Lee WL, Chen HL, Tsai TY, Lai IC, Chang WC, et al. (2009) Risk factors for peripheral intravenous catheter infection in hospitalized patients: a prospective study of 3165 patients. American journal of infection control 37(8): 683-686.

18. Veenstra DL, Saint S, Sullivan SD (1999) Cost-effectiveness of antisepticimpregnated central venous catheters for the prevention of catheterrelated bloodstream infection. Jama 282(6): 554-560.

19. Tagalakis V, Kahn SR, Libman M, Blostein M (2002) The epidemiology of peripheral vein infusion thrombophlebitis: a critical review. The American journal of medicine. 113(2): 146-151.

20. Vonberg RP, Gastmeier P (2007) Hospital-acquired infections related to contaminated substances. The Journal of hospital infection 65(1): 15-23.

21. Mattner F, Gastmeier P (2004) Bacterial contamination of multiple-dose vials: a prevalence study. American journal of infection control 32(1): 12-16.

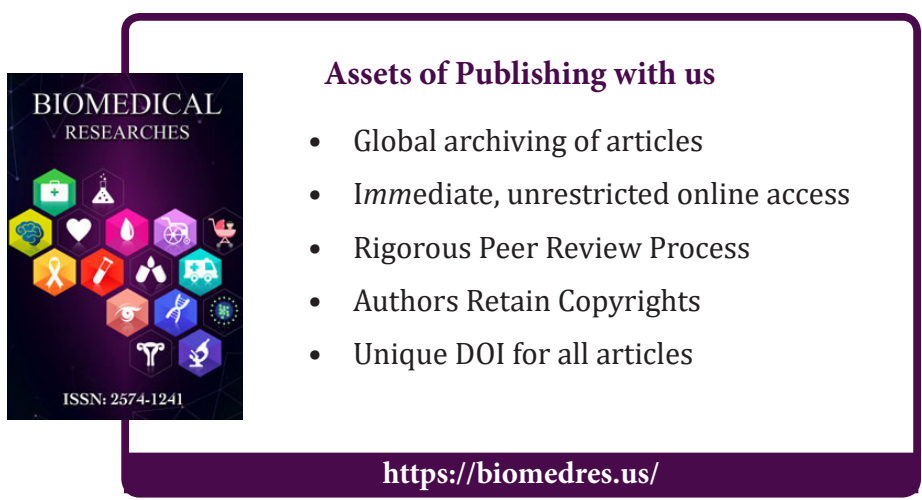

